\title{
Realização de testes rápidos de sífilis em gestantes por enfermeiros da atenção básica
}

Rapid syphilis tests in pregnant women by primary care nurses

Realización de exámenes rápidos de sífilis en gestantes por enfermeros de la atención primaria

\section{Bruna Britto Pereira ${ }^{\mathrm{I}}$, Cristiano Pinto dos Santos ${ }^{\mathrm{II}}$, Giovana Calcagno Gomes ${ }^{\mathrm{III}}$}

Resumo: Objetivo: conhecer de que forma os enfermeiros da atenção básica realizam os testes rápidos para sífilis em gestantes. Método: pesquisa qualitativa realizada em um município do sul do Brasil. Os dados foram coletados em 2018 por entrevistas semiestruturadas e submetidos à Análise de Conteúdo. Resultados: referiram que a doença pode ser assintomática, mas tem três estágios. Citaram como sintomas uma ferida vaginal que some e após aparecem manchas no corpo. A doença pode causar no recém-nascido má-formação. Houve desconhecimento acerca da doença. Notificam os casos positivos e iniciam imediatamente o tratamento da gestante. Ressaltaram a não adesão dos parceiros ao tratamento. Conclusão: destaca-se o importante papel do enfermeiro na realização do pré-natal e do teste rápido de sífilis. Observa-se que são necessárias ações de educação continuada melhorando os indicadores da doença no país.

Descritores: Enfermeiras de saúde da família; Sífilis congênita; Sorodiagnóstico da sífilis; Estratégia saúde da família; Atenção primária à saúde

Abstract: Objective: to find out how primary care nurses perform rapid tests for syphilis in pregnant women. Method: this is a qualitative research conducted in a municipality in southern Brazil. Data were collected in 2018 by semi-structured interviews and submitted to Content Analysis. Results: thee nurses reported that the disease can be asymptomatic, but it has three stages. The symptoms mentioned were a vaginal wound that disappears and after that spots appear on the body. The disease can cause malformation in the newborn. The disease was unknown. When they noticed positive cases, they immediately began treatment of the pregnant woman. They emphasized the non-adherence of partners to treatment. Conclusion: the nurse's important role in prenatal and rapid syphilis testing is highlighted. Continuing education actions are needed to improve the disease indicators in the country.

Keywords: Family health nurses; Congenital syphilis; Syphilis serodiagnosis; Family health strategy; Primary health care

\footnotetext{
I Enfermeira. Faculdade de Desenvolvimento do Rio Grande do Sul - FADERGS. Canoas, Rio Grande do Sul, Brasil. brunabritto94@hotmail.com https://orcid.org/0000-0002-2936-4647

II Enfermeiro. Doutor em Enfermagem. Universidade da Campanha. Bagé, Rio Grande do Sul, Brasil. enfcristiano.ps@hotmail.com https://orcid.org/0000-0001-8298-0652

III Enfermeira. Doutora em Enfermagem. Universidade Federal de Rio Grande - FURG. Rio Grande, Rio Grande do Sul, Brasil. giovanacalcagno@furg.br https://orcid.org/0000-0002-2464-1537
} 
Realização de testes rápidos de sífilis em gestantes por enfermeiros da atenção básica | 2

Resumen: Objetivo: conocer de qué forma los enfermeros de la atención primaria realizan los exámenes rápidos para sífilis en gestantes. Método: es una investigación cualitativa realizada en un municipio del sur de Brasil. Los datos fueron recogidos en 2018 por entrevistas semi-estructuradas y sometidos al Análisis de Contenido. Resultados: reportaron que la enfermedad puede ser asintomática, pero tiene tres etapas. Como síntomas, citaron una herida vaginal que desaparece y después aparecen manchas en el cuerpo. La enfermedad puede causar mala formación en el recién nacido. Se desconocía acerca de la enfermedad. Notificaron casos positivos e iniciaron inmediatamente el tratamiento de la gestante. Resaltaron la no adherencia de los compañeros al tratamiento. Conclusión: se destaca el importante papel del enfermero en la realización del prenatal y del examen rápido de sífilis. Se observa que son necesarias acciones de educación continuada mejorando los indicadores de la enfermedad en el país.

Palabras clave: Enfermeras de salud de la familia; Sífilis congénita; Serodiagnóstico de la sífilis; Estrategia salud de la familia; Atención primaria a la salud

\section{Introdução}

A Sífilis é um agravo de saúde de passível eliminação, desde que a gestante infectada pela bactéria Treponema Pallidum seja identificada e tratada o mais precocemente possível. Por ser uma doença de notificação compulsória, deve ser verificado e notificado o caso de todo recémnascido $(\mathrm{RN})$ vivo ou natimorto filho de mãe com sífilis. A principal função da notificação de doenças é fornecer uma base para implementação de políticas de saúde pública que promovam, protejam e controlem a saúde da população. O número de casos no Brasil aumentou entre 2014 e 2015 em 32,7\% para sífilis adquirida por transmissão sexual, 20,9\% na gestação e 1,9\% quando transmitida da mãe para o bebê. ${ }^{1}$

A Sífilis Congênita (SC) constitui-se um evento sentinela para acompanhamento da Atenção Primária em Saúde (APS). No Brasil, um estudo revelou que a prevalência de soropositividade para sífilis foi de 0,89\%, correspondendo a cerca de 26.700 gestantes/ano. ${ }^{2}$ Em base hospitalar esses índices são recorrentes, principalmente em mulheres na faixa-etária dos 20 aos 34 anos, com baixa escolaridade e mais vulneráveis socialmente. ${ }^{3}$

Apesar de se tratar de uma doença de fácil prevenção verificam-se falhas no funcionamento da rede de atenção básica, pois parte das gestantes infectadas não são adequadamente tratadas. São infectadas por seus parceiros, mantendo a cadeia de transmissão e, 
consequentemente, as altas taxas de sífilis. Falhas no aconselhamento, dificuldades para acessar testes, tratamento não realizado no local do diagnóstico e nenhum acompanhamento foram os principais obstáculos identificados. ${ }^{4}$

A Sífilis Gestacional (SG) tem importância significativa para a saúde pública, devido ao seu impacto durante a gravidez. Ela pode afetar o desenvolvimento do feto e do RN, aumentando a suscetibilidade ao aborto, parto prematuro, malformações esqueléticas, meningite e pneumonia. ${ }^{5}$ Uma das medidas de prevenção cabíveis ao enfermeiro é a realização do Teste Rápido para Sífilis em cada trimestre gestacional. Eles possibilitam a tomada de medidas de tratamento há tempo, evitando assim, a SC, prevenindo a Transmissão-Vertical.

Estudo realizado no nordeste brasileiro apontou que suas unidades básicas tinham profissionais treinados para realizar testes rápidos e esses estavam disponíveis. No entanto, em nenhuma das unidades foram realizados durante as consultas pré-natais. As que realizaram o teste convencional, Venereal Disease Research Laboratory (VDRL) referiram atraso na entrega dos resultados. Nenhuma das unidades básicas investigadas coletava material biológico diariamente, o que dificultava o acesso ao diagnóstico. Verificou-se que ao comparecerem para receberem os resultados do teste VDRL, a orientação que obtinham do aconselhamento restringia-se a informálas sobre os resultados do exame e a necessidade de tratar seus parceiros sexuais. ${ }^{4}$

No pré-natal, o teste para diagnosticar SG deve ser realizado logo no primeiro trimestre de gestação. O enfermeiro deverá realizar a solicitação do VDRL quantitativo em torno das 19 semanas de gestação. ${ }^{6}$ Apesar dos protocolos estabelecidos pelo Ministério da Saúde e a disponibilidade de diagnóstico e tratamento da sífilis nos serviços de saúde responsáveis pelo pré-natal, a eliminação da SC permanece um desafio. Enfatiza-se como importante para os profissionais de saúde, gestores e população em geral melhorar a qualidade do pré-natal com inclusão e corresponsabilidade dos parceiros sexuais e o uso de métodos como testes diagnósticos rápidos. ${ }^{7}$ 
Realização de testes rápidos de sífilis em gestantes por enfermeiros da atenção básica | 4

Nesse contexto, tem-se como questão de pesquisa: como os enfermeiros da atenção básica realizam os testes rápidos de sífilis em gestantes? Deste modo, teve-se como objetivo: conhecer de que forma os enfermeiros da atenção básica realizam os testes rápidos para sífilis em gestantes.

\section{Método}

Trata-se de uma pesquisa qualitativa, exploratória e descritiva a partir da qual foi possível observar as condutas do profissional enfermeiro frente à realização dos testes rápidos de sífilis nos trimestres gestacionais. Foi realizada em dez Estratégias Saúde da Família (ESF) de um município do sul do Brasil. As unidades foram definidas de forma aleatória. O município conta com 18 unidades de ESF. Cada unidade possui uma enfermeira.

Participaram do estudo dez enfermeiros atuantes nessas unidades há pelo menos um ano realizando o pré-natal. Os que aceitaram participar do estudo assinaram o Termo de Consentimento Livre e Esclarecido.

Os dados foram coletados nos meses de setembro e outubro de 2018, por meio de entrevistas semiestruturadas. Os participantes foram questionados acerca da forma como seguem o protocolo do Ministério da Saúde para a realização dos testes rápidos para sífilis em gestantes. Foram convidadas a participar do estudo verbalmente pelas pesquisadoras e realizadas no seu turno de trabalho em uma sala para garantir privacidade. Duraram cerca de 40 minutos, sendo áudio-gravadas e transcritas. Após os dados foram submetidos à Análise de conteúdo. ${ }^{8}$ Primeiramente, foi realizada uma leitura flutuante para estruturar os dados e após foram submetidos à exploração do material e tratamento, no qual foram estudados e discutidos a partir de autores estudiosos da temática.

Foram respeitadas as Resoluções 466/2012 e 510/2016 do Conselho Nacional de Saúde. O projeto foi aprovado pelo Comitê de Ética em Pesquisa sob o parecer $n^{\circ} 2.799 .661$ (CAAE: 
5 | Pereira BB, Santos CP, Gomes GC

94794518.0.0000.5340). A data da aprovação foi no dia 03 de agosto de 2018. Os participantes da pesquisa foram identificados pela letra “E”, seguida do número de realização das entrevistas.

\section{Resultados}

Foram entrevistadas 10 enfermeiras com idades entre 25 e 50 anos de idade. Cinco eram solteiras, quatro casadas e uma divorciada. Atuavam na ESF entre um e 26 anos. Realizavam o pré-natal: entre 17 anos (duas), 14 anos (duas), nove anos (uma), cincos anos (três) e um ano (duas). A análise dos dados gerou como categorias: Protocolo seguido pelos enfermeiros da atenção básica na realização dos testes rápidos para sífilis em gestante e Condutas das enfermeiras frente ao teste rápido com resultado positivo para sífilis.

\section{Protocolo seguido pelos enfermeiros da atenção básica na realização dos testes rápidos para sífilis em gestantes}

As participantes referiram que realizam os testes rápidos para Sífilis em todos os trimestres gestacionais durante o pré-natal em toda gestante e que o mesmo é obrigatório e que orientam acerca do teste que está realizando.

Sim. Nós realizamos o teste rápido de sifilis nas gestantes durante o pré-natal, nos três trimestres, de gestação. (E2)

Sim, a gente faz três testes rápidos, no primeiro, segundo e terceiro trimestre. Informamos para a gestante o que ela está realizando. (E5)

Sim. Eu realizo o teste rápido nos três trimestres, primeiro, segundo e terceiro. (E9)

Sim. Nós aqui realizamos no primeiro, segundo e terceiro trimestre de rotina. (E10)

No município, as gestantes são primeiramente atendidas em uma unidade de referência e após o diagnóstico da gravidez e realização do primeiro teste rápido essas são referenciadas para 
Realização de testes rápidos de sífilis em gestantes por enfermeiros da atenção básica | 6

seus bairros. Essa unidade de referência serve de apoio para orientações, sanar dúvidas e acompanhamento da gestante junto com os profissionais de saúde. Servem, também, como porta de entrada de gestantes de outros municípios da região.

A maioria das pacientes a gente faz duas vezes. No segundo e no terceiro trimestre, pois a porta de entrada é a Unidade Referência. Ela já vem para nós, com o primeiro teste realizado. (E1)

Sim, os testes são realizados na Unidade de Referência. Elas já realizam os testes rápidos e já são encaminhadas para a unidade do seu bairro com os testes rápidos realizados. (E6)

Eu faço o teste no momento do acolhimento. Elas já chegam ao posto com o teste realizado. Então, eu faço no primeiro momento e a cada trimestre. (E7)

Normalmente, ela já vem com os primeiros testes rápidos realizados. Dando positivo, ela já é notificada de que tem que fazer o tratamento lá no posto de referência. (E8)

As principais orientações veiculadas pelos enfermeiros às gestantes foram sobre a importância da realização do teste rápido de sífilis, do início precoce do tratamento e dos prejuízos que essa ocasiona no feto.

Pelos prejuízos que traz para o bebê. As más formações congênitas. A importância do tratamento e quanto mais precoce for feito o teste será possivel identificar o diagnóstico. (E3)

A importância da identificação do contágio. A transmissão para o feto correndo riscos de aborto, más-formações. (E10)

\section{Condutas das enfermeiras frente ao teste rápido com resultado positivo para sífilis}

Frente ao teste rápido de sífilis positivo as enfermeiras referiram fazer a notificação do caso e iniciar imediatamente o tratamento. É solicitado um VDRL quantitativo para confirmar o diagnóstico.

A nossa conduta seguindo o protocolo, realizamos o teste, dando positivo, já notificamos e no mesmo dia já realizamos o tratamento. (E1) 
Imediatamente nós já tratamos. Não se espera o confirmatório do VDRL quantitativo. É tratado com a penicilina benzatina. Fazemos três semanas de aplicações, depois solicitamos $o$ VDRL. (E3)

Apenas uma das participantes ressaltou que após o teste rápido de sífilis positivo, o tratamento não é iniciado na hora. Referiu que a gestante deverá esperar o resultado confirmatório do VDRL quantitativo para iniciar o tratamento.

Quando o teste dá positivo eu não trato, eu espero fazer o VDRL quantitativo. Quando vem confirmatório dou início o tratamento semanal com 2.400 de benzetacil. (E9)

Duas participantes relataram que a sífilis em gestante é tratada como terciária, iniciando o tratamento com 2.400.000 UI de penicilina benzatina por semana durante um período de três semanas, totalizando 7.200.000 UI.

A gente trata como terciária. Então, são três semanas de tratamento. No momento que eu fiz o teste rápido e deu positivo. Ela já vai fazer a primeira dose da medicação. (E2)

Nós tratamos como sífilis terciária no caso para liquidar. Três semanas na corrida. Onde se faz o controle mensal, solicitando o VDRL, para melhor acompanhamento. (E5)

Destacaram ser comum a não adesão dos parceiros ao tratamento junto com a gestante, possibilitando assim a reinfecção da mesma, aumentando as chances da transmissão vertical.

Tem casos em que o parceiro se negou a tratar. Fomos atrás da gestante e ela acabou perdendo o feto porque ela se recontaminou. (E3)

Tratar parceiro é bem mais difícil. Nós temos que correr atrás ou então elas dizem: Estou separada. Não sei quem é o pai. É bastante complicado o tratamento. (E5)

Acontece muito. Elas fazerem todo o tratamento e o marido ficar de ir e não aparecer. (E8)

\section{Discussão}

Verificou-se que os testes rápidos para sífilis em gestantes, são realizados nos três trimestres da gestação. Estudo apontou que metade dos casos de mães com sífilis (48,4\%) iniciaram o acompanhamento no primeiro trimestre de gestação. Porém, a maioria das gestantes apresentou 
Realização de testes rápidos de sífilis em gestantes por enfermeiros da atenção básica $\mid 8$

diagnóstico tardio. ${ }^{9}$ Em Fortaleza, o diagnóstico de sífilis ocorreu em $75,4 \%$ casos durante a gestação. Sendo que $86,1 \%$ receberam o diagnóstico entre o segundo e terceiro trimestre. ${ }^{10-11}$

Os testes rápidos são realizados durante a consulta, por meio da coleta de uma amostra de sangue, sendo possível o diagnóstico em até 20 minutos. A introdução do teste rápido no prénatal possibilita uma melhor cobertura de triagem para sífilis na gravidez, permitindo o diagnóstico e o tratamento imediato na gestante. ${ }^{12}$ A eliminação global da SC exigirá, no entanto, melhorar o acesso ao rastreio e o tratamento precoce da sífilis, monitorizar clinicamente todas as mulheres e crianças diagnosticadas com sífilis, a gestão do parceiro e reduzir a prevalência da sífilis na população em geral por meio da expansão dos testes rápidos. ${ }^{13}$

A implementação da rede cegonha pelo ministério da saúde, trouxe mudanças no atendimento à gestante, por parte dos profissionais de saúde, pela oferta dos testes rápidos para rastreio de sífilis e HIV (Vírus da Imunodeficiência Humana) nas unidades de atenção básica. O teste rápido de sífilis deve ser feito na primeira consulta do pré-natal, no primeiro trimestre de gestação, no início do terceiro trimestre $\left(28^{\circ}\right.$ semana $)$ e, no momento do parto ou aborto, independentemente de exames anteriores. ${ }^{11}$ Destaca-se o papel do enfermeiro no contato direto aos pacientes, na realização dos testes rápidos, identificação dos sinais e sintomas da doença, acompanhamento e oferta de orientações à família. ${ }^{14}$

O pré-natal é uma ferramenta importante no diagnóstico, orientação e acompanhamento da gestante na detecção da SG, visando à sua profilaxia precoce, evitando a infecção do RN. ${ }^{15}$ Em locais com recursos limitados, a triagem de mulheres grávidas para sífilis usando testes de diagnóstico rápido treponêmico simples (RDTs) é uma ferramenta fundamental na prevenção da SC, pois permitem uma maior cobertura de rastreio e a administração no mesmo dia do tratamento. ${ }^{16}$

O diagnóstico precoce da SC é o pré-requisito necessário para o tratamento oportuno. Se o diagnóstico de sífilis ocorrer nos dois primeiros trimestres da gravidez pode-se reduzir significativamente o risco de seus resultados adversos. ${ }^{17} \mathrm{O}$ aumento constante no número de 
casos de sífilis na população em geral pode ser atribuído ao aumento da cobertura de testagem, redução do uso de preservativos, resistência dos profissionais de saúde na administração da Penicilina na Atenção Básica, desabastecimento mundial da Penicilina, entre outros. ${ }^{18}$ Como existem muitos obstáculos para o desenvolvimento de uma vacina eficaz contra o T. pallidum, a triagem pré-natal universal e o tratamento adequado para a mãe e o RN continuam sendo a estratégia mais eficaz para a prevenção da transmissão da sífilis de mãe para filho. ${ }^{19}$

A qualificação profissional, a sensibilização e a padronização da conduta dos profissionais de saúde são necessárias. Oferecer apoio aos profissionais de saúde em suas práticas clínicas por meio de um processo de supervisão pode contribuir para a adoção das diretrizes recomendadas e para a promoção de cuidados com a saúde. ${ }^{4}$ Pressupõe-se que a enfermeira que realiza pré-natal de baixo risco, na unidade básica de saúde, deve buscar o conhecimento acerca das infecções sexualmente transmissíveis, sobretudo dos problemas que as mesmas fomentam para o feto. A falta de entendimento e convicção do assunto perante a gestante pode passar insegurança na consulta de pré-natal.

Há situações em que a sífilis pode ser assintomática, dificultando o diagnóstico, favorecendo a proliferação do Treponema pallidum. Tal fato atrasa o início do tratamento e aumenta as chances da bactéria ultrapassar a barreira placentária, acometendo o feto. A sífilis pode causar efeitos deletérios para a mãe e o feto se não identificada e tratada precocemente. ${ }^{14}$

Estudo que investigou a fragilidade do tratamento na estratégia saúde da família também mostrou que frente ao teste rápido com resultado positivo para sífilis os enfermeiros notificam o caso e iniciam imediatamente o tratamento. ${ }^{20}$ Foi destacado, também, a não adesão do parceiro ao tratamento junto com a gestante, possibilitando sua reinfecção, aumentando as chances da transmissão vertical da doença.

Considera-se importante a concordância do parceiro em realizar o tratamento da sífilis junto com a gestante como forma eficaz para combater a doença. O tratamento durante o pré- 
Realização de testes rápidos de sífilis em gestantes por enfermeiros da atenção básica | 10

natal pode ser ineficaz em até $14 \%$ dos casos e ainda há probabilidade de reinfecção caso o parceiro não tenha feito o tratamento corretamente. O mesmo deve ser tratado mesmo quando apresentam VDRL negativo, com dose única de penicilina benzatina 2.400 .000 UI via intramuscular. Quando o resultado for positivo, o tratamento é o mesmo da sífilis terciaria. ${ }^{21}$

\section{Conclusão}

O estudo contemplou conhecer de que forma os enfermeiros da atenção básica seguem o protocolo para a realização dos testes rápidos para sífilis em gestantes. Verificou-se estes são realizados nos três trimestres da gestação. As orientam que sua realização auxilia no diagnóstico da doença e os riscos dessa para o RN.

Frente a um resultado positivo para sífilis elas realizam a notificação do caso e iniciam imediatamente o tratamento da gestante. Foi destacado, também, ser comum a não adesão do parceiro ao tratamento, possibilitando assim a reinfecção da mesma, aumentando as chances da transmissão vertical. Destaca-se o importante papel do enfermeiro na realização do pré-natal e realização o mais precocemente possível do teste rápido, informando o diagnóstico e iniciando o tratamento da gestante e seu parceiro sexual imediatamente.

Para isso é necessária à capacitação dos enfermeiros acerca da doença e do Protocolo do Ministério da Saúde para realização do Teste rápido para Sífilis. No entanto, concluiu-se que há falhas na educação continuada desses profissionais. Essas comprometem a prevenção da doença, seu diagnóstico e tratamento bem como o acompanhamento das gestantes infectadas, seus parceiros e recém-nascidos.

Em relação à prática assistencial acredita-se importante a capacitação de todos os enfermeiros da atenção básica quanto à realização dos testes rápidos para detecção da sífilis em gestantes, minimizando seus efeitos, qualificando a realização do pré-natal. O estudo teve como limitações ter sido realizado em um único município. Novas pesquisas devem ser realizadas 
acerca da temática na busca de estratégias para diminuir o número de casos de sífilis, principalmente entre as gestantes e recém-nascidos.

\section{Referências}

1. Garbin AJÍ, Martins RJ, Belila NM, Exaltação SM, Garbin CAS. Reemerging diseases in Brazil: sociodemographic and epidemiological characteristics of syphilis and its under-reporting. Rev Soc Bras Med Trop. 2019;52:e20180226:1-4. doi: 10.1590/0037-8682-0226-2018

2. Cunha ARC, Merchan-Hamann E. Sífilis em parturientes no Brasil: prevalência e fatores associados, 2010 a 2011. Rev Panam Salud Publica [Internet]. 2015 [acesso em 2018 set 20];38(6):479-86. Disponível em: https://www.scielosp.org/article/rpsp/2015.v38n6/479-486/pt/

3. Domingues RMM, Leal MC. Incidência de sífilis congênita e fatores associados à transmissão vertical da sífilis: dados do estudo nascer no Brasil. Cad Saúde Pública. 2016;32(6):1-12. doi: 10.1590/0102311X00082415

4. Rocha AFB, Araújo MAL, Miranda AE, Leon RGP, Silva Junior GB, Vasconcelos LDPG. Management of sexual partners of pregnant women with syphilis in northeastern Brazil - a qualitative study. BMC Health Serv Res. 2019;19(1):65. doi: 10.1186/s12913-019-3910-y

5. Souza JM, Giuffrida R, Ramos APM, Morceli G, Coelho CH, Rodrigues MVP. Mother-to-child transmission and gestational syphilis: spatial-temporal epidemiology and demographics in a Brazilian region. PLoS Negl Trop Dis. 2019;13(2):e0007122. doi:10.1371/journal.pntd.0007122

6. Stafford IA, Berra A, Minard CG, Fontenot V, Kopkin RH, Rodrigue E, et al. Challenges in the contemporary management of syphilis among pregnant women in New Orleans, LA. Infect Dis Obstet Gynecol. 2019;Vol 2019:2613962. doi: 10.1155/2019/2613962

7. Silva Neto SE, Silva SSBE, Sartori AMC. Syphilis in pregnancy, congenital syphilis, and factors associated with mother-to-child transmission in Itapeva, São Paulo, 2010 to 2014. Rev Soc Bras Med Trop. 2018;51(6):819-26. doi: 10.1590/0037-8682-0377-2017

8. Bardin L. Análise de conteúdo. Lisboa: Edições 70; 2009.

9. Lafetá KRG, Martelli Júnior H, Silveira MF, Paranaíba LMR. Sífilis materna e congênita, subnotificação e difícil controle. Rev Bras Epidemiol. 2016;19(1):63-74. doi: 10.1590/19805497201600010006

10. Cardoso ARP, Araújo MAL, Cavalcante MS, Frota MA, Melo SP. Análise dos casos de sífilis gestacional e congênita nos anos de 2008 a 2010 em Fortaleza, Ceará, Brasil. Ciênc Saúde Colet. 2018;23(2):563-74. doi: 10.1590/1413-81232018232.01772016 
Realização de testes rápidos de sífilis em gestantes por enfermeiros da atenção básica | 12

11. Machado I, Silva VAN, Pereira RMS, Guidoreni CG, Gomes MP. Diagnóstico e tratamento de sífilis durante a gestação: desafio para as enfermeiras? Saúde Pesqui [Internet]. 2018;11(2):249-55. doi 10.17765/2176-9206.2018v11n2p249-255

12. Araújo EC, Monte PCB, Haber ANCA. Avaliação do pré-natal quanto à detecção de sífilis e HIV em gestantes atendidas em uma área rural do estado do Pará, Brasil. Rev Pan Amaz Saúde [Internet]. 2018; 09(1):33-9. doi: 10.5123/s2176-62232018000100005

13. Korenromp EL, Rowley J, Alonso M, Mello MB, Wijesooriya NS, Mahiané SG, et al. Global burden of maternal and congenital syphilis and associated adverse birth outcomes-estimates for 2016 and progress since 2012. PLoS One. 2019;14(2):e0211720. doi: 10.1371/journal.pone.0211720

14. Ministério da Saúde (BR), Secretaria de Vigilância em Saúde, Departamento de DST, Aids e Hepatites Virais. Protocolo clínico e diretrizes terapêuticas para atenção integral às pessoas com infecções sexualmente transmissíveis [Internet]. Brasília (DF): Ministério da Saúde; 2015 [acesso em 2018 ago 15]. Disponível

em: http://bvsms.saude.gov.br/bvs/publicacoes/protocolo_clinico_diretrizes_terapeutica_atencao_integral_pe ssoas_infeccoes_sexualmente_transmissiveis.pdf

15. Rodrigues VLR, Oliveira FM, Afonso TM. Sífilis congênita na perspectiva de um desafio para a saúde pública. Congresso Internacional de Enfermagem - CIE; 2017 maio 09-12; Aracaju, SE. Anais. (Congresso Int Enferm [Internet]. 2017 [acesso em 2018 jun 23];1(1):1-4. Disponível em https://eventos.set.edu.br/index.php/cie/article/view/5958).

16. Langendorf C, Lastrucci C, Sanou-Bicaba I, Blackburn K, Marie-Hortense K, Crucitti T. Dual screen and confirm rapid test does not reduce overtreatment of syphilis in pregnant women living in a nonvenereal treponematoses endemic region: a field evaluation among antenatal care attendees in Burkina Faso. Sex Transm Infect. 2018;95(6):402-4. doi: 10.1136/sextrans-2018-053722

17. Braccio S, Sharland M, Ladhani S. Prevention and treatment of mother-to-child transmission of syphilis. Curr Opin Infect Dis. 2016 Jun;29(3):268-74. doi: 10.1097/QCO.0000000000000270

18. Ministério da Saúde (BR). Boletim epidemiológico - sífilis [Internet]. Brasília (DF): Ministério da Saúde; 2017 [acesso em 2018 set 22]. Disponível em: http://www.aids.gov.br/pt-br/pub/2017/boletimepidemiologico-de-sifilis-2017

19. Zhang $\mathrm{X}, \mathrm{Yu} \mathrm{Y}$, Yang H, Xu H, Vermund SH, Liu K. Surveillance of maternal syphilis in China: pregnancy outcomes and determinants of congenital syphilis. Med Sci Monit. 2018 Oct 29;24:7727-35. doi: 10.12659/MSM.910216

20. Silva AS, Magalhães Sobrinho DDT, Nascimento MRL, Rodrigues TL, Vasconcelos LMO. Sífilis em gestantes: investigação da fragilidade do tratamento na estratégia saúde da família. X Mostra Científica da Farmácia. 2016; Quixadá, CE. Anais. (Mostra Cient Farm [Internet]. 2016 [acesso em 2018 ago 15];3(1). 
Disponível

em:

http://publicacoesacademicas.unicatolicaquixada.edu.br/index.php/mostracientificafarmacia/article/view/1270).

21. Ministério da Saúde (BR). Protocolo clínico e diretrizes terapêuticas para prevenção da transmissão vertical de HIV, sífilis e hepatites virais [Internet]. 2017 [acesso em 2018 set 15]. Disponível em: http://www.aids.gov.br/pt-br/pub/2015/protocolo-clinico-e-diretrizes-terapeuticas-para-prevencao-datransmissao-vertical-de-hiv

Editor Científico Chefe: Cristiane Cardoso de Paula

Editor Científico: Tânia Solange Bosi de Souza Magnago

\section{Autor correspondente}

Bruna Britto Pereira

E-mail: brunabritto94@hotmail.com

Endereço: Alameda das Corticeiras 165, Bairro Mato Grande. Canoas, Rio Grande do Sul.

CEP: 92320177

\section{Contribuições de Autoria}

\section{1 - Bruna Britto Pereira}

Concepção ou desenho do estudo/pesquisa, análise e/ou interpretação dos dados, revisão final com participação crítica e intelectual no manuscrito.

\section{2 - Cristiano Pinto dos Santos}

Concepção ou desenho do estudo/pesquisa; análise e/ou interpretação dos dados, revisão final com participação crítica e intelectual no manuscrito.

\section{3 - Giovana Calcagno Gomes}

Concepção ou desenho do estudo/pesquisa, análise e/ou interpretação dos dados, revisão final com participação crítica e intelectual no manuscrito.

\section{Como citar este artigo}

Pereira BB, Santos CP, Gomes GC. Realização de testes rápidos de sífilis em gestantes por enfermeiros da atenção básica. Rev. Enferm. UFSM. 2020 [Acesso em: Ano Mês Dia]; vol.10 e82: 1-13 DOI:https://doi.org/10.5902/2179769240034 